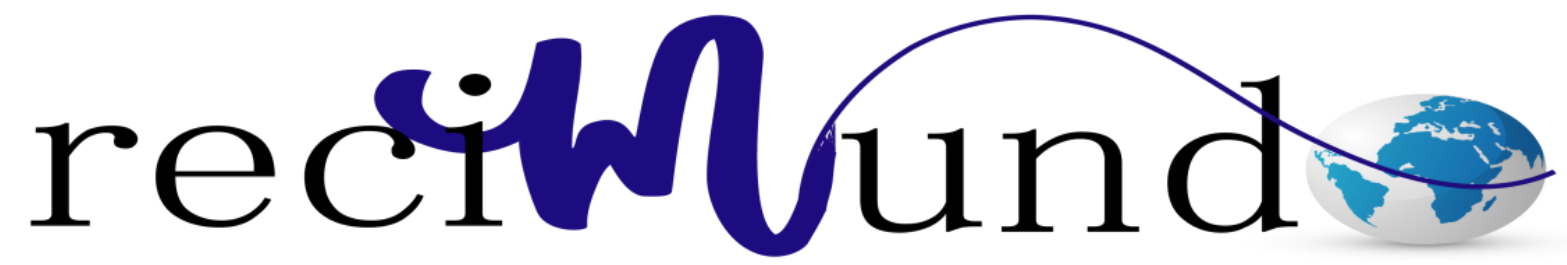

Revista Científica Mundo de la Investigación y el Conocimiento

Lucia Estefanía Osorio Vera a; María Estefanía Arce Dueñas ${ }^{\text {b; }}$ Jefferson Leonardo León García ${ }^{\text {c}}$; Mónica Viviana Macancela Feijoo d

Uso de métodos anticonceptivos en adolescentes entre las edades de 15 a 17 años en la maternidad Mariana de Jesús en el período 2013-2014

Use of contraceptive methods in adolescents between the ages of 15 and 17 in the Mariana de Jesús maternity ward in the 2013-2014 period

Revista Científica Mundo de la Investigación y el Conocimiento. Vol. 3 núm. 4., diciembre, ISSN: 2588-073X, 2019, pp. 138-149

DOI: $10.26820 /$ recimundo/3.(4).diciembre.2019.138-149

URL: http://recimundo.com/index.php/es/article/view/656

Código UNESCO: 3205 Medicina Interna

Tipo de Investigación: Artículo de Revisión

(C) RECIMUNDO; Editorial Saberes del Conocimiento, 2019

Recibido: 15/09/2019

Aceptado: 23/11/2019

Publicado: 30/12/2019

Correspondencia: luciaoso14@hotmail.com
a. Médico; Investigador Independiente; Guayaquil, Ecuador; luciaoso14@ hotmail.com
b. Médico; Investigador Independiente; Guayaquil, Ecuador; doctoraestefi@gmail.com
c. Médico; Investigador Independiente; Guayaquil, Ecuador; jeffo.leo.grc@gmail.com
d. Médico; Investigador Independiente; Guayaquil, Ecuador; monicamacancelafeijoo@ gmail.com 


\section{Uso de métodos anticonceptivos en adolescentes entre las edades de 15 a 17 años en la maternidad Mariana de Jesús en el período 2013-2014}

Vol. 3, núm. 4., (2019)

Lucia Estefania Osorio Vera; María Estefanía Arce Dueñas; Jefferson Leonardo León García; Mónica Viviana Macancela Feijoo

\section{RESUMEN}

La presente investigación tiene como objetivo orientar a los adolescentes sobre las complicaciones de no usar métodos anticonceptivos. El diseño de esta investigación es de tipo acción participativa, seleccionando la muestra de acuerdo a la utilidad de la respectiva investigación como se da a continuación y por último, la acumulación de datos que se efectuó en un solo momento determinado. La población total del estudio fueron las 300 adolescentes de consulta externa de la Maternidad Mariana De Jesús en el periodo 2013-2014 La muestra estuvo conformada por 30 pacientes que se seleccionó aleatoriamente debido a la necesidad de investigación que se tuvo para realizar el estudio determinado. La metodología que se utilizó fue la aplicación de encuestas a las pacientes. El problema de la presente investigación se centra en el desconocimiento de los adolescentes sobre la importancia de los métodos anticonceptivos en las relaciones sexuales, para la prevención de un embarazo o enfermedad de transmisión sexual. Los adolescentes expresan que dentro de sus hogares no existe una comunicación de los padres hacia los hijos sobre temas de sexualidad, ya que piensan que tocar estos temas incentivará a los adolescentes a iniciar su sexualidad a temprana edad, sin los debidos cuidados y protecciones necesarios para la salud. La autora en observancia a lo investigado bibliográficamente tiene como objetivo dar a conocer o difundir el uso de métodos anticonceptivos en adolescentes.

Palabras Claves: Orientación; Conocimiento; Métodos anticonceptivos; Adolescentes. 
Uso de métodos anticonceptivos en adolescentes entre las edades de 15 a 17 años en la maternidad Mariana de Jesús en el período 2013-2014

Vol. 3, núm. 4., (2019)

Lucia Estefania Osorio Vera; María Estefanía Arce Dueñas; Jefferson Leonardo León García; Mónica Viviana Macancela Feijoo

\section{ABSTRACT}

Introduction: This research aims to provide guidance to adolescents about the complications of not using contraception. The design of this research is participatory action type, selecting the sample according to the utility of the respective investigation as given below and finally the accumulation of data which took place in a single given time. The total population of the study were 300 adolescents outpatient Maternity Mariana de Jesus in 2013-2014 The sample consisted of 30 patients who were randomly selected due to the need for research to be had for the given study. The methodology used was the application of surveys to patients. The problem of this research focuses on the lack of adolescents about the importance of contraception in sexual relations to prevent pregnancy or sexually transmitted disease. Teenagers express that in their homes there is no communication of the fathers to their children about sexuality issues because they think that playing these issues will encourage teenagers to start their sexuality at an early age, without proper care and protection necessary for the health. The author investigated in compliance with the bibliographically aims to publicize or promote the use of contraceptive methods in adolescents.

Keywords: Orientation; Knowledge; Contraceptive methods; Teenagers. 


\section{Uso de métodos anticonceptivos en adolescentes entre las edades de 15 a 17 años en la maternidad Mariana de Jesús en el período 2013-2014}

Vol. 3, núm. 4., (2019)

Lucia Estefania Osorio Vera; María Estefanía Arce Dueñas; Jefferson Leonardo León García; Mónica Viviana Macancela Feijoo

\section{Introducción.}

En el mundo actualmente se ha incrementado el índice de embarazos no deseados que generan abandono escolar en los adolescentes, se ha elevado el contagio de enfermedades de transmisión sexual, encontrando, así como problema principal el poco conocimiento de métodos anticonceptivos los cuales son de mucha utilidad para evitar que ocurran todas estas situaciones, estos métodos impiden una posible fecundación en adolescentes que mantienen relaciones sexuales. (Langer, 2002) (Morales Díaz, Solanelles Rojas, \& Mora González, 2013)

Estos problemas pueden repercutir significativamente no solo en la salud también en lo social llevando a los adolescentes muchas veces a tomar malas decisiones como la práctica de un aborto, usar drogas, ingerir alcohol que llevan muchas veces al suicidio, situaciones muy lamentables que se viven día a día en colegios y hogares del mundo. (Núñez-Urquiza, Hernández-Prado, \& García-Barrios, 2003) (Ariza Riaño, Valderrama Sanabria, \& Ospina Díaz, 2014)

La falta de comunicación con sus padres, los malos consejos de sus amistades, la influencia de la televisión con mensajes distorsionados sobre sexualidad han llevado a la mayoría de adolescentes a tener su primera relación sexual a temprana edad, muchas veces por curiosidad, los cuales muchas veces tienen varias parejas sexuales. (Mendoza Tascón, Claros Benítez, \& Peñaranda Ospina, 2016) (Quintero Rondón \& Rojas Betancur, 2015) 
Uso de métodos anticonceptivos en adolescentes entre las edades de 15 a 17 años en la maternidad Mariana de Jesús en el período 2013-2014

Vol. 3, núm. 4., (2019)

Lucia Estefania Osorio Vera; María Estefanía Arce Dueñas; Jefferson Leonardo León García; Mónica Viviana Macancela Feijoo

Esta investigación se realizó en la Maternidad Mariana de Jesús en el sur - oeste de Guayaquil en el cual se encuestará y orientará en la consulta externa sobre métodos anticonceptivos a las adolescentes y se conseguirá concientizar sobre las consecuencias que pueden tener malas decisiones con respecto a su sexualidad para lo cual se debe conocer sobre estos métodos de planificación familiar.

\section{Metodología.}

La presente investigación se efectuó en el Hospital Maternidad Marianita de Jesús MSP del Ecuador en la ciudad de Guayaquil.

\section{Universo}

Todas las pacientes que utilizan el servicio de consulta externa de la Maternidad Mariana de Jesús entre las edades 15 a 17 años.

Muestra

Se incluirán en el estudio las pacientes que han acudido a consulta externa por primera vez para aplicarse un método anticonceptivo en la Maternidad Mariana de Jesús en el periodo 2013-2014.

Se excluirán de la muestra las pacientes que se les ha realizado cesárea y parto y que posteriormente solicitan post cirugía y post parto un método anticonceptivo.

Diseño de investigación 


\section{Uso de métodos anticonceptivos en adolescentes entre las edades de 15 a 17 años en la maternidad Mariana de Jesús en el período 2013-2014}

Vol. 3, núm. 4., (2019)

Lucia Estefania Osorio Vera; María Estefanía Arce Dueñas; Jefferson Leonardo León García; Mónica Viviana Macancela Feijoo

La Investigación es de tipo descriptivo que permitió evaluar la información recolectada para la investigación., transversal por que se estudia a los sujetos en un mismo momento, no experimental; es decir no mantuvimos bajo control a la muestra investigada.

\section{Análisis de la información}

Para el análisis de los datos obtenidos se realizó una labor estadística descriptiva en base a porcentajes y frecuencia de cada una de las variables evaluadas y de sus posibles combinaciones.

Los datos obtenidos se registraron en el formulario de estudio (Anexos), cuyos datos del formulario del proyecto han sido revisados y supervisados por el asesor metodológico de la tesis.

Los datos fueron procesados en computadora y se utilizó el procesador de textos, cuyos resultados se presentan en gráficos: circulares y columnas; cada uno con sus respectivos porcentajes; lo cual permite mostrar los resultados de una manera más clara y sencilla.

\section{Viabilidad}

El desarrollo de la tesis es viable porque se cuenta con la asesoría y orientación del tutor legal designado, además de contar con los recursos necesarios tanto económicos, humanos, materiales, bibliográficos de mucha importancia para realizar una investigación de este tipo.

Todos los objetivos planteados y las soluciones propuestas buscan la identificación real del no uso de métodos anticonceptivos en adolescentes mujeres de 15 a 17 años de la Maternidad Mariana de Jesús, ayudando así a la prevención, detección oportuna y disminución de 
Uso de métodos anticonceptivos en adolescentes entre las edades de 15 a 17 años en la maternidad Mariana de Jesús en el período 2013-2014

Vol. 3, núm. 4., (2019)

Lucia Estefania Osorio Vera; María Estefanía Arce Dueñas; Jefferson Leonardo León García; Mónica Viviana Macancela Feijoo

enfermedades para que los índices de embarazos no deseados y enfermedades de transmisión sexual desciendan.

\section{Resultados.}

\section{Cuadro $\mathbf{N}^{\circ}$ 1. Edad}

\begin{tabular}{|c|c|c|c|}
\hline $\mathbf{N}^{\mathbf{0}}$ & Detalle & Cantidad & $\%$ \\
\hline 1 & 15 años & 20 & $67 \%$ \\
\hline 2 & 16 años & 7 & $23 \%$ \\
\hline 3 & 17 años & 3 & $10 \%$ \\
\hline 4 & Otros & 0 & $0 \%$ \\
\hline \multicolumn{2}{|c|}{ Total } & $\mathbf{3 0}$ & $\mathbf{1 0 0 \%}$ \\
\hline
\end{tabular}

Análisis: De la población encuestada, el 67\% expresa que tiene una edad de 15 años, un 23\% refiere que tiene una edad de 16 años y un $10 \%$ expresa que tiene una edad de 17 años.

Cuadro $\mathbf{N}^{\circ}$ 2. Sexo

\begin{tabular}{|l|l|c|c|}
\hline $\mathbf{N}^{\mathbf{0}}$ & Detalle & Cantidad & \% \\
\hline 1 & Femenino & 30 & $100 \%$ \\
\hline \multicolumn{2}{|c|}{ Total } & $\mathbf{3 0}$ & $\mathbf{1 0 0 \%}$ \\
\hline
\end{tabular}

Análisis: Los datos tabulados en la presente tabla nos muestran que el 100\% de la población encuestada expresa que son de sexo femenino.

Cuadro $\mathbf{N}^{\mathrm{o}}$ 3. ¿Con quién vive?

\begin{tabular}{|c|c|c|c|}
\hline $\mathbf{N}^{\mathbf{0}}$ & Detalle & Cantidad & $\mathbf{\%}$ \\
\hline 1 & Mamá & 4 & $13 \%$ \\
\hline 2 & Papá & 8 & $27 \%$ \\
\hline
\end{tabular}




\section{Uso de métodos anticonceptivos en adolescentes entre las edades de 15 a 17 años en la maternidad Mariana de Jesús en el período 2013-2014}

Vol. 3, núm. 4., (2019)

Lucia Estefania Osorio Vera; María Estefanía Arce Dueñas; Jefferson Leonardo León García;

Mónica Viviana Macancela Feijoo

\begin{tabular}{|c|c|c|c|}
\hline 3 & Tíos & 0 & $0 \%$ \\
\hline 4 & Padres & 18 & $60 \%$ \\
\hline 5 & Otros & 0 & $0 \%$ \\
\hline & Total & $\mathbf{3 0}$ & $\mathbf{1 0 0} \%$ \\
\hline
\end{tabular}

Análisis: Los datos graficados en el presente cuadro estadístico nos refleja que el 60\% expresa que vive con sus padres, un $27 \%$ manifiesta que vive solo con sus papás y un $13 \%$ manifiesta que vive con sus mamás.

Cuadro $\mathbf{N}^{\circ}$ 4. ¿Sabe usted que es un método anticonceptivo?

\begin{tabular}{|c|c|c|c|}
\hline $\mathbf{N}^{\mathbf{0}}$ & Detalle & Cantidad & $\mathbf{\%}$ \\
\hline 1 & $\mathrm{Si}$ & 16 & $53 \%$ \\
\hline 2 & $\mathrm{No}$ & 14 & $47 \%$ \\
\hline \multicolumn{2}{|c|}{ Total } & $\mathbf{3 0}$ & $\mathbf{1 0 0 \%}$ \\
\hline
\end{tabular}

Análisis: De la población encuestada, el 53\% afirma que si sabe sobre qué es un método anticonceptivo y un $47 \%$ expresa que no tiene conocimiento sobre los métodos anticonceptivos.

Cuadro $\mathbf{N}^{0}$ 5. ¿Usted está de acuerdo en utilizar métodos anticonceptivos para el control de la natalidad?

\begin{tabular}{|l|c|c|c|}
\hline $\mathbf{N}^{\mathbf{0}}$ & Detalle & Cantidad & $\mathbf{\%}$ \\
\hline 1 & $\mathrm{Si}$ & 13 & $43 \%$ \\
\hline 2 & $\mathrm{No}$ & 17 & $57 \%$ \\
\hline \multicolumn{2}{|c|}{ Total } & $\mathbf{3 0}$ & $\mathbf{1 0 0 \%}$ \\
\hline
\end{tabular}

Análisis: Los datos tabulados en la presente tabla nos muestran que el 57\% de la población encuestada expresa que no está de acuerdo en que los métodos anticonceptivos se deben usar para controlar la natalidad, un $43 \%$ afirma que si deben ser considerados como control de la natalidad. 
Uso de métodos anticonceptivos en adolescentes entre las edades de 15 a 17 años en la maternidad Mariana de Jesús en el período 2013-2014

Vol. 3, núm. 4., (2019)

Lucia Estefania Osorio Vera; María Estefanía Arce Dueñas; Jefferson Leonardo León García; Mónica Viviana Macancela Feijoo

Cuadro $\mathrm{N}^{\circ}$ 6. De estos anticonceptivos ¿Cuál es el que más ha oído nombrar?

\begin{tabular}{|l|c|c|c|}
\hline $\mathbf{N}^{\mathbf{0}}$ & Detalle & Cantidad & $\mathbf{\%}$ \\
\hline 1 & Condón & 20 & $67 \%$ \\
\hline 2 & Diu & 0 & $0 \%$ \\
\hline 3 & Anticonceptivos orales/inyectables & 7 & $23 \%$ \\
\hline 4 & Método del ritmo & 3 & $10 \%$ \\
\hline 5 & Ligadura de trompas & 0 & $0 \%$ \\
\hline 6 & Vasectomía & 0 & $0 \%$ \\
\hline 7 & Otros & 0 & $0 \%$ \\
\hline \multicolumn{2}{|c|}{ Total } & $\mathbf{3 0}$ & $\mathbf{1 0 0} \%$ \\
\hline
\end{tabular}

Análisis: Los datos graficados en el presente cuadro estadístico nos refleja que el 57\% de la población encuestada expresa que el condón es el anticonceptivo que más han oído hablar, mientras que el 23\% expresa que han escuchado de los anticonceptivos orales/inyectables y un $10 \%$ han escuchado del método del ritmo.

Cuadro $\mathbf{N}^{\mathbf{0}}$ 7. ¿Ha utilizado métodos anticonceptivos?

\begin{tabular}{|l|l|c|c|}
\hline $\mathbf{N}^{\mathbf{0}}$ & Detalle & Cantidad & \% \\
\hline 1 & $\mathrm{Si}$ & 14 & $47 \%$ \\
\hline 2 & No & 16 & $53 \%$ \\
\hline \multicolumn{2}{|c|}{ Total } & $\mathbf{3 0}$ & $\mathbf{1 0 0 \%}$ \\
\hline
\end{tabular}

Análisis: De la población encuestada, el 53\% indica que no ha utilizado métodos anticonceptivos y un $47 \%$ señala que si ha utilizado métodos anticonceptivos.

Cuadro $\mathbf{N}^{0}$ 8. ¿En el colegio usted recibe información sobre métodos anticonceptivos?

\begin{tabular}{|l|c|c|c|}
\hline $\mathbf{N}^{\mathbf{0}}$ & Detalle & Cantidad & \% \\
\hline 1 & $\mathrm{Si}$ & 10 & $33 \%$ \\
\hline 2 & $\mathrm{No}$ & 20 & $67 \%$ \\
\hline \multicolumn{2}{|c|}{ Total } & $\mathbf{3 0}$ & $\mathbf{1 0 0 \%}$ \\
\hline
\end{tabular}




\section{Uso de métodos anticonceptivos en adolescentes entre las edades de 15 a 17 años en la maternidad Mariana de Jesús en el período 2013-2014}

Vol. 3, núm. 4., (2019)

Lucia Estefania Osorio Vera; María Estefanía Arce Dueñas; Jefferson Leonardo León García; Mónica Viviana Macancela Feijoo

Análisis: Los datos tabulados en la presente tabla nos muestran que el $67 \%$ de la población encuestada indica que no recibe información sobre los métodos anticonceptivos en el colegio, mientras que el 33\% señala que si recibe este tipo de información dentro de la institución.

Cuadro No 9. ¿Considera usted que los metodos anticonceptivos son abortivos?

\begin{tabular}{|l|c|c|c|}
\hline $\mathbf{N}^{\mathbf{0}}$ & Detalle & Cantidad & \% \\
\hline 1 & $\mathrm{Si}$ & 19 & $63 \%$ \\
\hline 2 & $\mathrm{No}$ & 11 & $37 \%$ \\
\hline \multicolumn{2}{|c|}{ Total } & $\mathbf{3 0}$ & $\mathbf{1 0 0 \%}$ \\
\hline
\end{tabular}

Análisis: Los datos graficados en el presente cuadro estadístico nos refleja que el 63\% de la población encuestada indica que si consideran los métodos anticonceptivos como abortivos, mientras que el 37\% señala que no lo consideran como abortivo.

Cuadro $\mathbf{N}^{\mathbf{0}}$ 10. ¿Le gustaría recibir información sobre métodos anticonceptivos?

\begin{tabular}{|l|c|c|c|}
\hline $\mathbf{N}^{\mathbf{0}}$ & Detalle & Cantidad & $\mathbf{\%}$ \\
\hline 1 & $\mathrm{Si}$ & 30 & $100 \%$ \\
\hline 2 & $\mathrm{No}$ & 0 & $0 \%$ \\
\hline \multicolumn{2}{|c|}{ Total } & $\mathbf{3 0}$ & $\mathbf{1 0 0 \%}$ \\
\hline
\end{tabular}

Análisis: De la población encuestada, el 100\% expresa que si desearía recibir información sobre los métodos anticonceptivos.

\section{Conclusiones.}

- A través de la investigación se detectó que existe falta de comunicación de los padres con los hijos, por lo cual estos adoptan inadecuadas e iniciación precoz de la sexualidad. 
Uso de métodos anticonceptivos en adolescentes entre las edades de 15 a 17 años en la maternidad Mariana de Jesús en el período 2013-2014

Vol. 3, núm. 4., (2019)

Lucia Estefania Osorio Vera; María Estefanía Arce Dueñas; Jefferson Leonardo León García; Mónica Viviana Macancela Feijoo

- Los adolescentes expresan que tienen poco conocimiento sobre el uso de los métodos anticonceptivos, quedando expuestos a contraer enfermedades de transmisión sexual, embarazo no deseado o aborto.

- Se observa que dentro de la institución no se realizan campañas dirigidas a los estudiantes sobre la sexualidad.

La mayoría de los adolescentes reciben influencia a través de los medios de comunicación y amistades, lo cual causa en ellos conductas inadecuadas, desinterés de las consecuencias e irresponsabilidad.

\section{Bibliografía.}

Ariza Riaño, N., Valderrama Sanabria, M., \& Ospina Díaz, J. (2014). Caracterización del embarazo adolescente en dos ciudades de Boyacá, Colombia. Revista Virtual Universidad Católica del Norte, 42(1), 154-163.

Langer, A. (2002). El embarazo no deseado: impacto sobre la salud y la sociedad en América Latina y el Caribe. Revista Panamericana de Salud Pública, 11(3), 192-205.

Mendoza Tascón, L., Claros Benítez, D., \& Peñaranda Ospina, C. (2016). Actividad sexual temprana y embarazo en la adolescencia: estado del arte. Revista chilena de obstetricia y ginecología, 81(3), 243-253.

Morales Díaz, E., Solanelles Rojas, A., \& Mora González, S. (2013). Embarazo no deseado en alumnas universitarias. Revista Cubana de Medicina Militar, 42(2), 153-163.

Núñez-Urquiza, R., Hernández-Prado, B., \& García-Barrios, C. (2003). Embarazo no deseado en adolescentes, y utilización de métodos anticonceptivos posparto. Salud Pública de México, 45(1), 93-102.

Quintero Rondón, A., \& Rojas Betancur, H. (2015). El embarazo a temprana edad, un análisis desde la perspectiva de madres adolescentes. Revista Virtual Universidad Católica del Norte, 44(1), 222-237. 
Uso de métodos anticonceptivos en adolescentes entre las edades de 15 a 17 años en la maternidad Mariana de Jesús en el período 2013-2014 Vol. 3, núm. 4., (2019)

Lucia Estefania Osorio Vera; María Estefanía Arce Dueñas; Jefferson Leonardo León García; Mónica Viviana Macancela Feijoo

RECONOCIMIENTO-NOCOMERCIAL-COMPARTIRIGUAL
CC BY-NC-SA
ESTA LICENCIA PERMITE A OTROS ENTREMEZCLAR, AJUSTAR Y CONSTRUIR A PARTIR DE SU OBRA CON FINES NO COMERCIALES,
SIEMPRE Y CUANDO LE RECONOZCAN LA AUTORÍA Y SUS NUEVAS CREACIONES ESTÉN BAJO UNA LICENCIA CON LOS MISMOS
TÉRMINOS.

